\title{
The Diagnostic Value of Monocyte Chemoattractant Protein-1, Compared with Procalcitonin, C-reactive Protein, and Lactate in Bacteremia Estimation for Patients with Febrile Neutropenia
}

\author{
İlker Ödemişş ${ }^{1 *}$, Şükran Köse² ${ }^{2}$, Süheyla Serin Senger² ${ }^{2}$ İlkay Akbulut² ${ }^{2}$ Didem \\ Çelik ${ }^{2}$,
}

\begin{abstract}
1. Infectious Diseases and Clinical Microbiology Clinic, Başkent University, Turkey
2. Infectious Diseases and Clinical Microbiology Clinic, Sağlık Bilimleri University, Tepecik Training and Research Hospital, Turkey
\end{abstract}

\begin{abstract}
Bacteremia in the febrile neutropenic patients significantly increases the mortality. It takes a long time to complete the blood culture for the diagnosis of bacteremia. Therefore, quick and specific markers are needed for the prediction of bacteremia. The purpose of this study are to compare the diagnostic value of lactate, procalcitonin, $C$-reactive protein (CRP) and monocyte chemoattractant protein-1 (MCP-1) levels in a patient with febrile neutropenia, and to evaluate its usefulness in predicting bacteremia. This study was designed to be prospective case-control study. Forty-eight patients and forty control cases aged 18 years or older who were monitored between May 2016 and May 2017 were included in the study. P-value as $<0.05$ was accepted to be significant. Significantly increased values were determined by the level of inflammatory markers of patients compared to the control group. The highest diagnostic odds ratio were found to be in MCP-1. For patients with febrile neutropenia, CRP (83.3\%), and MCP1 (81.2\%) were the most sensitive markers while lactate (85.0\%), MCP-1 (75\%), and procalcitonin (75\%) were the most specific markers. CRP was the only beneficial biomarker in the estimation of bacteremia. No significant results were observed for any biomarker for the prediction of the gram positive/negative discrimination of bacteria in the blood culture. We believe that CRP, MCP-1, and lactate levels can be taken into consideration for diagnosis, and CRP can be beneficial in the estimation of bacteremia.
\end{abstract}

Keywords: biomarkers, procalcitonin, bacteremia, Febrile neutropenia, monocyte chemoattractant protein-1 Received: 28 ${ }^{\text {th }}$ June 2020; Accepted: 12 th October 2020; Published: $26^{\text {th }}$ October 2020

\footnotetext{
* Corresponding author: İlker Ödemiş, Infectious Diseases and Clinical Microbiology Clinic, Başkent University, Adana, Turkey. E-mail: ilkerodemis2014@gmail.com
} 


\section{Introduction}

Cancer is one of the greatest health concerns of today. The World Health Organization estimates that 14.1 million people develop cancer every year (1). According to a study carried out in the United Kingdom, it is believed that one in every two people will develop cancer at some point in their lives (2). One of the most considerable complications of cytotoxic chemotherapy given during cancer treatment is febrile neutropenia (FN). The infections that develop in $\mathrm{FN}$ are the leading causes of mortality (3). Blood culture is the gold standard for the diagnosis of bacteremia. However, time required for blood culture to produce a result can be quite long, and the sensitivity of the blood culture is known as low (4). There is a need for quick, and reliable biomarkers for the estimation of bacteremia or early diagnosis of bacteremia in FN.

C-reactive protein (CRP) levels rise in many various cases of inflammation, thus, it has a low specificity for infections (5). The results of studies show that the procalcitonin test has high specificity for infections $(3,5)$. However, the sensitivity and specificity change depending on the cut-off value of procalcitonin (6). The lactate level can increase in plasma in certain cases such as tissue hypoxia, toxemia, metabolic diseases, and sepsis. Chemokines are secreted against bacterial, viral, parasitic, and mycobacterial infections. Monocyte chemo-attractant protein-1 (MCP-1) is an important chemokine ensuring the migration of monocytes and macrophages to the inflammation area during inflammation (7). There are numerous studies regarding the diagnostic values of MCP-1, procalcitonin, $\mathrm{CRP}$, and lactate levels in FN (3-5,8-10). However, there a consensus has not been reached regarding the benefits of biomarkers in the diagnosis of patients and estimation of bacteremia.

The purpose of this study was to compare the diagnostic value of lactate, procalcitonin, CRP, and MCP-1 levels in patient with $\mathrm{FN}$, and to evaluate its usefulness in predicting bacteremia.

\section{Materials and Methods}

This study is designed to be a prospective case-control study. Forty-eight FN patients aged 18 years or older who were monitored between May 2016 and May 2017 at the infectious diseases and clinical microbiology service or hematology service were included in the study. Forty people who had neutrophil count as $<500 / \mathrm{mm} 3$, who had $<38.0^{\circ} \mathrm{C}$ body temperature, and who had no focal infection were recruited as the control group. Only the first attack of the patients who had more than one FN attack during the study was taken into consideration. Patients who were undergoing antibiotic, antiviral, and antifungal treatment were excluded.

Informed consent was obtained from all individual participants included in the study. The institutional ethics review board approved the study protocol (Approval no: 21/1, date: 15 March 2016). All procedures performed in the study were in accordance with the ethical standards of the institutional and/or national research committee and with 1975 (revised in 2000) Helsinki declaration and its later amendments or comparable ethical standards. No funding was received for this research.

Upon determining fever, we collected at least two sets of blood culture (used both aerobic and anaerobic bottles) from each patient. The blood culture were analyzed under automatized system (BacT/ALERT, Biomériux, ABD) in the microbiology laboratory. Positive blood culture is defined as observing at least one pathogenic microorganism in at least one blood culture, or the observation of skin flora microorganisms in at least two blood cultures from patients with at least one of the following clinical findings: fever, trembling, and hypotension. Blood cultures were incubated for seven days in a device before negative assessment. 
Biomarker tests were performed in the biochemistry laboratory. Serum procalcitonin level, lactate, and CRP levels were analyzed ECLIA (electrochemiluminescence immunoassay) (Roche DiagnosticsGmbH, Mannheim, Germany) set within Cobas e analyzer, automatized blood gas device (Siemens RAPIDLab 1265) and nephelometric method (Immage Immunochemistry Systems CRP reagent, Beckman Coulter, ABD), respectively. References ranges of lactate, $\mathrm{PCT}$, and CRP were 0.2-3.0 $\mathrm{mmol} / \mathrm{L}, 0-0.1 \mathrm{ng} / \mathrm{mL}$, $0-0.8 \mathrm{mg} / \mathrm{dL}$ in healthy humans.

The samples taken for MCP-1 analysis were centrifuged, the serum was separated, and the samples were preserved at $-80^{\circ} \mathrm{C}$. Serum MCP-1 level was analyzed by micro-enzyme-linked immunosorbent assay method and with PICOKINE (Boster Biological Technology, Pleasanton, California, the USA) set manually according to the instructions of the manufacturing company. The sensitivity range was $15.6 \mathrm{pg} / \mathrm{ml}-1000 \mathrm{pg} / \mathrm{ml}$ and the sensitivity was $<1 \mathrm{pg} / \mathrm{ml}$ for MCP-1 set.

\section{Statistical analysis}

Medcalc 14 (Acacialaan 22, B-8400 Ostend, Belgium) and SPSS 22.0 (IBM Corporation, Armonk, New York, the United States) software packages were used for the analysis of the variables. The compliance of the data to normal distribution was assessed by the Shapiro-Wilk test while variance homogeneity was assessed by the Levene test. In the comparison of two independent groups according to quantitative data, the Independent-Samples T-test was used with Bootstrap results while the Mann-Whitney U test was performed with Monte Carlo results. In the comparison of categorical variables, the Pearson Chi-Square test was utilized by Monte Carlo Simulation method and Fisher Exact test was performed with exact results. Logistics regression test was performed by the Backward method in order to determine the reason and re- sult relation of categorical respond variable with explanatory variables in dual (diotome) categories. The quantitative variables were indicated as \pm std. (average deviation) and median range (min.-max.), and the categorical variables were shown as $\mathrm{n}(\%)$ in the tables. The variables were analyzed with $95 \%$ confidence level $(\mathrm{Cl})$ and p-value below 0.05 were accepted as significant.

\section{Results}

Of the 88 people who took part in the study, 48 $(54.5 \%)$ were from the febrile neutropenic patients group while $40(45.5 \%)$ belonged to the control group. The mean age of FN patients was $62.17 \pm 12.69$ years (age range: $28-94$ ), while the mean age of the control group was $62.40 \pm 14.30$ years (age range: 20-84). The percentage of females was $47.9 \%$ and $47.5 \%$ for the patients and control groups, respectively. The mean body mass indices (BMI) were $24.46 \pm 3.17 \mathrm{~kg}$ / $\mathrm{m} 2$ (range: $18.49-32.71$ ) and $23.98 \pm 3.16 \mathrm{~kg} / \mathrm{m} 2$ (range: 17.63-30.74) for the patient and control groups, respectively. There was no statistically significant difference between the two groups in terms of age, sex, and BMI ( $p>0.05)$.

The median value of CRP, procalcitonin, lactate, and MCP-1 was $16.30 \mathrm{mg} / \mathrm{dL}, 2.15 \mathrm{ng} / \mathrm{ml}, 1.45$ $\mathrm{mmol} / \mathrm{L}, 143.95 \mathrm{pg} / \mathrm{ml}$ for the patients, respectively. The median value of CRP, procalcitonin, lactate, and MCP-1 was $2.20 \mathrm{mg} / \mathrm{dL}, 0.15 \mathrm{ng} /$ $\mathrm{ml}, 1.10 \mathrm{mmol} / \mathrm{L}, 79.50 \mathrm{pg} / \mathrm{ml}$ for the control groups, respectively. The median value of the markers was higher in the patients group, and the difference was significant $(p<0.05)$.

The lowest statistical $p$-value was $\mathrm{p}<0.001$ for MCP-1, CRP, and procalcitonin. The markers with the highest sensitivity were CRP and MCP1 , and the markers with the highest specificity were lactate, MCP-1, and procalcitonin (Table 1) (Figure 1).

The highest diagnostic ratio was 13.8 for MCP$1>100.27 \mathrm{pg} / \mathrm{ml}(\mathrm{p}<0.001)(95 \% \mathrm{Cl})$, and the 
Table 1. The cut-off, AUC and p values of the CRP, PCT, lactate, and MCP-1 of the patient and control groups

\begin{tabular}{lcccc}
\hline Indicator $/$ & Patient & Control & \multirow{2}{*}{ AUC \pm Se. } & \multirow{2}{*}{ P Value } \\
\cline { 2 - 3 } Cut-Off value & $\mathbf{n}(\mathbf{\%})$ & $\mathbf{n}(\mathbf{\%})$ & & \\
\cline { 1 - 2 } CRP $(\mathrm{mg} / \mathrm{dL})$ & $8(16.7)$ & $28(70.0)^{* *}$ & $0.834 \pm 0.042$ & $<0.001^{*}$ \\
$\leq 7$ & $40(83.3)^{*}$ & $12(30.0)$ & & \\
$>\mathbf{>}$ & $12(25.0)$ & $30(75.0)^{* *}$ & $0.822 \pm 0.044$ & $<0.001^{*}$ \\
\hline PCT $(\mathrm{ng} / \mathrm{ml})$ & $36(75.0)^{*}$ & $10(25.0)$ & & \\
$\leq \mathbf{0 . 6}$ & & & & $0.006^{*}$ \\
$>\mathbf{0 . 6}$ & $26(54.2)$ & $34(85.0)^{* *}$ & $0.659 \pm 0.058$ & \\
\hline Lactate $(\mathrm{mmol} / \mathrm{L})$ & $22(45.8)^{*}$ & $6(15.0)$ & & \\
$\leq \mathbf{1 . 6}$ & & & & \\
$>\mathbf{1 . 6}$ & $9(18.8)$ & $30(75.0)^{* *}$ & $0.823 \pm 0.046$ & $<0.001^{*}$ \\
\hline MCP-1 $(\mathrm{pg} / \mathrm{ml})$ & $39(81.3)^{*}$ & $10(25.0)$ & & \\
$\leq \mathbf{1 0 0 . 2 7}$ & & & \\
$>\mathbf{1 0 0 . 2 7}$ & & & & \\
\hline
\end{tabular}

ROC (Receiver Operating Curve) Analysis (Honley\&Mc Nell - Youden index J) AUC: Area Under Curve Se: Standard error Sensitivity * Specificity ** (The optimal cut off values were derived from ROC curve analysis and the point with the highest sensitivity and specificity was the optimal predictive value), CRP: C-reactive protein, PCT: Procalcitonin, MCP-1: Monocyte chemo-attractant protein-1.

second highest odds ratio was 6.7 for procalcitonin $>0.6 \mathrm{ng} / \mathrm{ml}(\mathrm{p}=0.017)(95 \% \mathrm{Cl})($ Table 2$)$. Ten $(20.8 \%)$ of the patients demonstrated bacteremia. The distribution of the factors was as follows: three (30\%) Enterococcus faecalis, two (20\%) Pseudomonas aeruginosa, one (10\%) methicillin-resistant Staphylococcus aureus, one (10\%) methicillin-sensitive S.aureus, one (10\%) Escherichia coli, one (10\%) Enterobacter aerogenes, and one (10\%) Klebsiella pneumoniae. In the comparison of biomarker levels of the patients with and without bacteremia, it was found that only the CRP level of bacteremia patients showed a significantly higher value $(p=0.044)$ (Figure 2) (Table 3).
None of the markers were found beneficial for estimating the gram stain characteristic of bacteria in the blood culture $(p>0.05)$. There were no side effects due to biomarker tests and blood cultures.

\section{Discussion}

Obesity, sex, and age can be a risk factor for certain diseases. There have been studies which reported that age, sex, and BMI are not risk factors as regards to FN $(3,11)$. Similarly, there was no difference in our study between the two groups in terms of age, sex, and BMI.

Table 2. The diagnostic odds ratio for PCT and MCP-1

\begin{tabular}{lcccccc}
\hline & \multirow{2}{*}{ RC } & \multirow{2}{*}{ SEM } & \multirow{2}{*}{ P Value } & \multirow{2}{*}{ Odds Ratio } & \multicolumn{2}{c}{ \%95 Cl for Odds Ratio } \\
\cline { 5 - 7 } & & & & & LCL & UCL \\
\hline PCT $>\mathbf{0 . 6} \mathbf{~ n g} / \mathbf{m l}$ & -1.916 & 0.802 & 0.017 & 6.792 & 1.412 & 32.680 \\
\hline MCP-1 $>\mathbf{1 0 0 . 2 7} \mathbf{~ p g} / \mathbf{m l}$ & -2.628 & 0.685 & $<0.001$ & 13.845 & 3.617 & 52.999 \\
\hline Constant & 3.450 & 0.737 & $<0.001$ & & & \\
\hline
\end{tabular}

Multitiple Logistic Regression (Method = Backward Stepwise (Wald)) SEM: Standart Error Mean, RC: Regression Coefficient, LCL: Lower Confidence limit, UCL: Upper Confidence Limit, PCT: Procalcitonin, MCP-1: Monocyte chemo-attractant protein-1. 


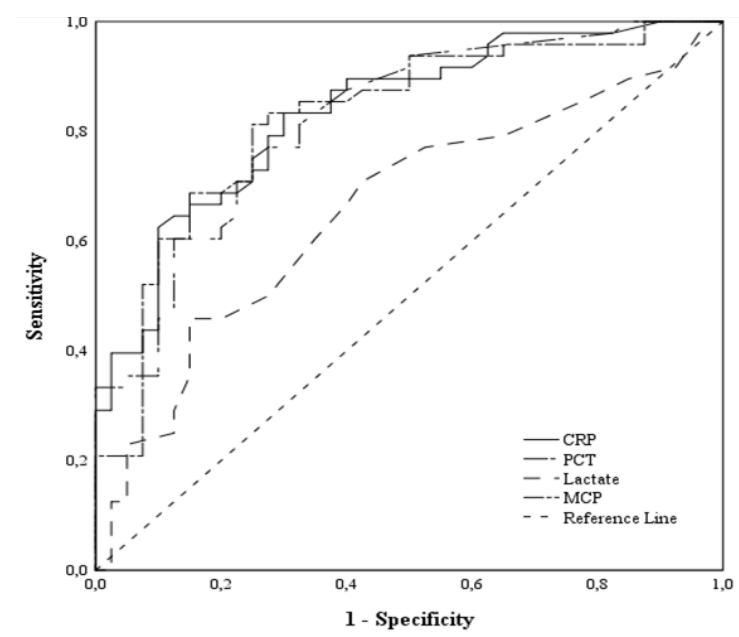

Fig. 1. The sensitivities and specificities of the markers with ROC curve chart, CRP: C-reactive protein, PCT: procalcitonin, MCP-1: monocyte chemo-attractant protein-1

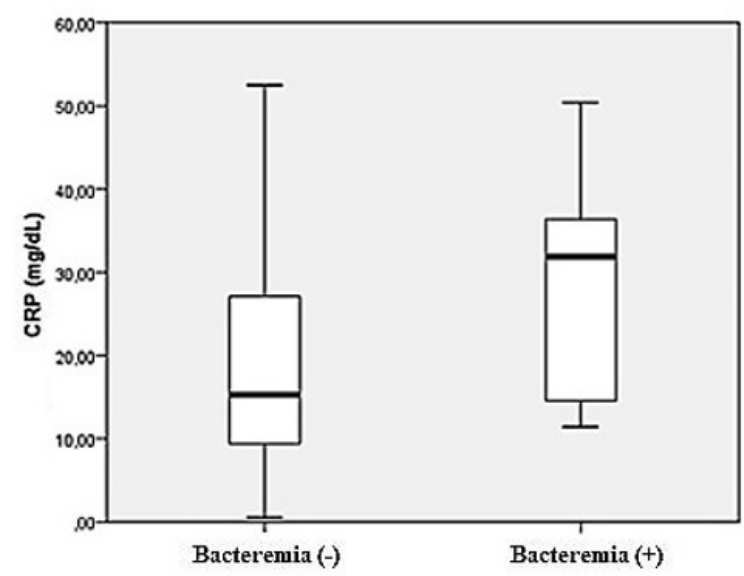

Fig. 2. The distribution of CRP in the bacteremic and non-bacteremic groups, CRP: c-reactive protein

Table 3. The comparison of the parameters of the bacteremic and non-bacteremic patients

\begin{tabular}{lccc}
\hline & \multicolumn{2}{c}{ Bacteremia } & \\
\cline { 2 - 4 } & $\begin{array}{c}\text { Positive } \\
(\mathbf{n}=\mathbf{1 0})\end{array}$ & $\begin{array}{c}\text { Negative } \\
(\mathbf{n}=\mathbf{3 8})\end{array}$ & \multirow{2}{*}{ P Value } \\
\cline { 2 - 4 } & Median (Min-Max) & Median (Min-Max) & $0.044^{*}$ \\
\hline CRP $(\mathbf{m g} / \mathbf{d L})$ & $31.9(11.4-50.4)$ & $15.25(0.5-52.5)$ & 0.266 \\
\hline PCT $(\mathbf{n g} / \mathbf{m l})$ & $2.75(0.4-75)$ & $2.15(0.09-60.9)$ & 0.617 \\
\hline Lactate $(\mathbf{m m o l} / \mathbf{L})$ & $1.75(0.6-3.2)$ & $1.4(0.6-6.4)$ & 0.757 \\
\hline MCP-1 $(\mathbf{p g} / \mathbf{m l})$ & $127.56(40.5-436.42)$ & $149.2(41.36-521.88)$ &
\end{tabular}

Mann Whitney U test (Monte Carlo) /Max.:Maximum - Min.: Minimum, CRP: C-reactive protein, PCT: Procalcitonin, MCP-1: Monocyte chemo-attractant protein-1. 
In the studies, CRP level showed an increase in FN $(3,4)$. Similar to other studies, ours revealed that the CRP values of FN patients were significantly high. It is believed that CRP can be beneficial for the exclusion of FN due to its high sensitivity.

Mato et al. (10) concluded that the increased lactate level of FN patients is related to septic shock development. Our study revealed that the difference between the lactate levels of the patient and control groups was statistically significant. Even though lactate was the most specific marker in our study, we believe that its low sensitivity limits its use in the diagnosis of FN.

Procalcitonin levels of febrile neutropenic patients significantly increased and procalcitonin levels can be applied for the early diagnosis of infection in neutropenic patients (12). Our results we obtained in compliance with other studies. We think that procalcitonin level can be beneficial in the early diagnosis of FN and its differentiation from other diseases that cause neutropenia.

Neuenschwander et al. (9) revealed that the MCP-1 level of FN patients who needed antibiotic replacement was significantly high and MCP-1 has a significant association with a 28day mortality. According to EL-Maghraby et al. (8), MCP-1 level increases in febrile neutropenic patients with local or systemic infection, and the level of MCP-1 in bacterial infections is higher in comparison with viral infections. We found that MCP-1 level was higher in the patient group compared with the control group. MCP-1 can be used in the early diagnosis and differentiation of FN due to its high sensitivity and specificity. However, we also believe that its high cost and the lack of an accepted cut-off value limit its practical use.

The bacteremia rate in $\mathrm{FN}$ is $14.1-21.7 \%$ (1214). The rates of gram positive bacteria in bacteremic FN patients were found to be $33-60 \%$ $(12,13,15)$. In this study, bacteremia and gram positive rates were found to comply with other studies. However, the rate of detecting enterococci in the blood culture was slightly higher than other studies in the literature $(14,16)$. This condition was thought to arise from the frequent use of urinary catheters at our hospital and the excessive use of quinolones and cephalosporin antibiotics in the society.

There are studies that conclude that biomarkers can be beneficial in the prediction of bacteremia in FN, while other authors report otherwise $(8,16,17)$. In our study, only CRP was found to be beneficial of four markers. We believe that taking CRP level into consideration can be helpful in the choice of empirical treatment.

Predicting the bacteria type in the blood culture before receiving the culture results may be beneficial when selecting antibiotics. Prat et al. (3) achieved significant results with procalcitonin, as regards to the prediction of gram negative bacteremia. However, there are other studies that obtained no significant result (18). Our study revealed that the markers are not beneficial in discriminating between gram-negative and positive microorganisms in the blood culture. Conflicting results between studies is thought to be associated with the variations in the distribution of bacteria in the blood culture or the use of different kits in the studies.

As a result, CRP was the most sensitive marker while lactate was the most specific marker. MCP-1 can be used in the diagnosis of FN due to its high sensitivity and specificity. The fact that CRP provided statistically significant results in the prediction of bacteremia was an important finding. We think that it will be useful for clinicians for prediction of prognosis of infectious disease in FN patients.

Though the literature contains many studies which separately examine the markers in patients with FN, the studies that compare all the four markers in nonfebrile neutropenic patients are limited in number. We believe that this will 
be a guiding study in terms of revealing the prominence of markers in FN and showing their benefits in clinical use. The inability to continually follow markers due to lack of funds limited our study. We believe that monitoring biomarkers at regular intervals may provide useful and beneficial information for survival and antibiotherapy revision of patients.

\author{
Abbreviations \\ MCP-1 - Monocyte chemo-attractant protein-1 \\ CRP - C-reactive protein \\ $\mathrm{FN}$ - Febrile neutropenia \\ ECLIA - Electrochemiluminescence immunoas- \\ say \\ CI - Confidence interval
}

\section{Authors Contributions}

İÖ (Conceptualization, methodology, software, validation, validation, investigation, resources, writing - original draft preparation, writing - review and editing, visualization, project administration)

ŞK (Data curation, methodology, validation, writing - original draft preparation, writing - review and editing, supervision)

SSS (Validation, formal analysis, writing - original draft preparation, writing - review and editing, supervision)

İA (Data curation, investigation, resources, validation, writing - original draft preparation, writing - review and editing)

DÇ (Software, data curation, validation, formal analysis, investigation, writing - original draft preparation, writing - review and editing)

\section{Conflict of Interest}

There are no conflicts of interest to declare with respect to this article.

\section{References}

1. McGuire S. World Cancer Report 2014. Geneva, Switzerland: World Health Organization, International Agency for Research on Cancer, WHO Press, 2015. Adv Nutr. 2016;7(2):418-9. DOI: 10.3945/an.116.012211

2. Ahmad AS, Ormiston-Smith N, Sasieni PD. Trends in the lifetime risk of developing cancer in Great Britain: comparison of risk for those born from 1930 to 1960. Br J Cancer. 2015;112(5):943-7. DOI: 10.1038/ bjc. 2014.606

3. Prat C, Sancho JM, Dominguez J, Xicoy B, Giménez $\mathrm{M}$, Ferrà $\mathrm{C}$, et al. Evaluation of procalcitonin, neopterin, C-reactive protein, IL-6 and IL- 8 as a diagnostic marker of infection in patients with febrile neutropenia. Leuk Lymphoma. 2008;49(9):1752-61. DOI: 10.1080/10428190802258956

4. Richter ME, Neugebauer S, Engelmann F, Hagel S, Ludewig K, La Rosée P, et al. Biomarker candidates for the detection of an infectious etiology of febrile neutropenia. Infection. 2016;44(2):175-86. DOI: $10.1007 /$ s15010-015-0830-6

5. Yang M, Choi SJ, Lee J, Lee DG, Kim YJ, Park YJ, et al. Serum procalcitonin as an independentdiagnostic markers of bacteremia in febrilepatients with hematologic malignancies. PLoS One. 2019;14(12):e0225765. DOI: 10.1371/journal.pone.0225765

6. Secmeer G, Devrim I, Kara A, Ceyhan M, Cengiz $\mathrm{B}$, Kutluk T, et al. Role of procalcitonin and CRP in differentiating a stable from a deteriorating clinical course in pediatric febrile neutropenia. J Pediatr Hematol Oncol 2007; 29: 107-11. DOI: 10.1097/ MPH.0b013e3180320b5b

7. Melgarejo E, Medina MÁ, Sánchez-Jiménez F, Urdiales JL. Monocyte chemoattractant protein-1: A key mediator in inflammatory processes. Int J Biochem Cell Biol 2009;41:998-1001. DOI: 10.1016/j.biocel.2008.07.018

8. El-Maghraby SM, Moneer MM, Ismail MM, Shalaby LM, El-Mahallawy HA. The diagnostic value of C-reactive protein, interleukin- 8 , and monocyte chemotactic protein in risk stratification of febrile neutropenic children with hematologic malignancies. J Pediatr Hematol Oncol. 2007;29(3):131-6. DOI: 10.1097/ MPH.0b013e3180308770

9. Neuenschwander LC, Bittencourt H, Ribeiro AFT, Teixeira AL, Teixeira MM, Teixeira JC, et al. Plasma levels of procalcitonin and eight additional inflammatory molecules in febrile neutropenic patients. Clinics (Sao Paulo). 2011;66(10):1699-705.

10. Mato AR, Luger S, Loren AW, Heitjan DF, Olson ER, Ujjani C, et al. Serum lactic acid (LA) as a predictor of septic shock in patients with hematologic malignancies (HM) who develop febrile neutropenia. Blood. 2008;112(11):666. DOI: 10.1182/blood. V112.11.666.666 
11. Collins JM, Fleming GF, Christ TN. Comparison of the incidence of febrile neutropenia in obese and normal weight breast cancer patients receiving myelosuppressive chemotherapy and prophylactic pegfilgrastim. J Oncol Pharm Pract. 2019;25(5):1112-8. DOI: $10.1177 / 1078155218776471$

12. Işlak Mutcalı S, Saltoğlu N, Balkan İI, Ozaras R, Yemişen M, Tabak F, et al. Early Changes of Mannose-Binding Lectin, H-Ficolin, and Procalcitonin in Patients with Febrile Neutropenia: A Prospective Observational Study. Turk J Haematol. 2016;33(4):30410. DOI: $10.4274 /$ tjh.2014.0385

13. Lubwama M, Phipps W, Najjuka CF, Kajumbula $\mathrm{H}$, Ddungu $\mathrm{H}$, Kambugu $\mathrm{H}$, et al. Bacteremia in febrile cancer patients in Uganda. BMC Res Notes. 2019;12(1):464. DOI: 10.1186/s13104-019-4520-9

14. Klastersky J, de Naurois J, Rolston K, Rapoport B, Maschmeyer G, Aapro M, et al. ESMO Guidelines Committee. Management of febrile neutropaenia: ESMO Clinical Practice Guidelines. Ann Oncol. 2016;27(5):111-8. DOI: 10.1093/annonc/mdw325

15. Carvalho AS, Lagana D, Catford J, Shaw D, Bak N.
Bloodstream infections in neutropenic patients with haematological malignancies. Infect Dis \& Health. 2020;25(1):22-9. DOI: 10.1016/j.idh.2019.08.006

16. Michel CS, Teschner D, Wagner EM, Theobald M, Radsak MP. Diagnostic value of sTREM-1, IL-8, PCT, and CRP in febrile neutropenia after autologous stem cell transplantation. Ann Hematol. 2017;96(12):2095101. DOI: $10.1007 / \mathrm{s} 00277-017-3128-1$

17. Lima SSS, Nobre V, de Castro Romanelli RM, Clemente WT, Da Silva Bittencourt HN, Melo ACM, et al. Procalcitonin-guided protocol is not useful to manage antibiotic therapy in febrile neutropenia: a randomized controlled trial. Ann Hematol. 2016;95(7):1169-76. DOI: $10.1007 / \mathrm{s} 00277-016-2639-5$

18. García de Guadiana-Romualdo L, Espa-ol-Morales I, Cerezuela-Fuentes P, Consuegra-Sánchez L, Hernando-Holgado A, Esteban-Torrella $\mathrm{P}$, et al. Value of lipopolysaccharide binding protein as diagnostic marker of infection in adult cancer patients with febrile neutropenia: comparison with C-reactive protein, procalcitonin, and interleukin 6. Support Care Cancer. 2015;23(7):2175-82. DOI: 10.1007/s00520-014-2589-1 